\title{
Curative effects of RF combined with DSA-guided ethanol sclerotherapy in venous malformations
}

\author{
XING WANG, JIAN MENG, JIE ZHANG, RUIHAN WU, JIANMIN GU, CUILING SHAO and KUN HAN \\ Department of Stomatology, The Central Hospital of Xuzhou, Xuzhou Clinical School \\ of Xuzhou Medical College, Xuzhou, Jiangsu 221009, P.R. China
}

Received January 29, 2016; Accepted September 19, 2016

DOI: $10.3892 /$ etm.2016.3804

\begin{abstract}
The aim of the study was to investigate the clinical effect of radiofrequency (RF) ablation combined with digital subtraction angiography (DSA)-guided anhydrous ethanol injection sclerotherapy in the treatment of high-return flow venous malformation (VM). Forty-one patients who were diagnosed as high-return flow VM with clinical and radiographic evidence were divided into the observation group $(n=19)$ and control group $(n=22)$ by random number table. All the patients received DSA-guided anhydrous ethanol injection sclerotherapy while the patients in the observation group were given RF ablation in addition. The clinical effect, treatment times and adverse effects of the two groups were analyzed. The effectiveness of the observation group was significantly higher than that of the control group (94.7 vs. $81.8 \%)$. Facial paralysis occurred in three patients $(13.6 \%)$ after ethanol injection in the control group, while the observation group had no facial nerve injury after treatment. The patients in the observation group had significantly fewer number of ethanol injections. RF can improve the efficacy of high-return flow VM and reduce the number of ethanol injections. The scheme is safe and effective, which is worth expanding in clinical practice.
\end{abstract}

\section{Introduction}

Venous malformations (VM) is the most common vascular malformation in clinical practice, and its incidence is $1 / 5,000-1 / 10,000$ with $40 \%$ in the head and neck (1). The most common treatment methods at present include surgical excision, freezing, laser, injection sclerotherapy and microwave irradiation $(2,3)$. The above methods are effective in treating low reflux VM with limited scope of lesion. However, there is

Correspondence to: Professor Jian Meng, Department of Stomatology, The Central Hospital of Xuzhou, Xuzhou Clinical School of Xuzhou Medical College, 199 South Jiefang Street, Xuzhou, Jiangsu 221009, P.R. China

E-mail: bqqq19778228@163.com

Key words: radiofrequency ablation, venous malformation, ethanol, sequential combined treatment a lack of effective means to treat with the deep and extensive high-return flow VM that are involved at multiple anatomical partitions. At present, the predominant way to treat with the high-return flow VM is comprehensive sequence therapy that focuses on digital subtraction angiography (DSA)-guided anhydrous ethanol injection sclerotherapy (4).

In the present study, we applied radiofrequency (RF) ablation in addition to the anhydrous ethanol injection sclerotherapy and achieved very good curative effects without any obvious adverse reactions.

\section{Patients and methods}

Patients. A total of 41 patients diagnosed with head and neck VM in the Department of Stomatology, Xuzhou Hospital Affiliated to Southeast University (Jiangsu, China) were enrolled in the study. Of the 41 patients, 15 were male and 26 female, with an age range of 5-61 years. The sites of lesions included: tongue, parotid, neck, cheek, lower eyelid, forehead, lips and throat. The average volume of tumor was $5.0 \times 2.0 \times 1.5-16.0 \times 12.0 \times 5.0 \mathrm{~cm}$. Inclusion criteria of the study were: i) Patients that were confirmed with type III, or type IV VM under image classification (5), and had received no treatment before surgery; ii) patients without history of serious cardiovascular and cerebrovascular disease and alcohol allergy; iii) patients that were confirmed with normal preoperative blood routine, liver function, renal function, electrocardiogram and chest X-ray examination; and iv) all of the enrolled patients were quite clear of the content of the study and provided written informed consent. The patients were randomly divided into the anhydrous ethanol injection $+\mathrm{RF}$ group (treatment group) and pure anhydrous ethanol injection group (control group). Table I presents the general information and the tumor diameter of the two groups of patients. After statistical comparison, it was found that the differences between the two groups were not statistically significant ( $P>0.05)$, with comparability. The study was approved by the Ethics Committee of the Central Hospital of Xuzhou. Written informed consent was obtained from all the participants or their families prior to the start of the study.

Treatment methods. Patients in the control group were treated using DSA-guided anhydrous ethanol injection sclerotherapy. Treatment was conducted under general or local anesthesia. 
Table I. Distribution of patients and control groups according to site of lesion.

Site of focus (case)

Gender (case)

Average age Diameter of tumor

Lower

Group Case Male Female (years, mean $\pm \mathrm{SD}) \quad(\mathrm{cm}$, mean $\pm \mathrm{SD})$ Tongue Parotid Cheek Lip Throat Forehead eyelid Neck

\begin{tabular}{llllllllllllll}
\hline Treatment & 19 & 6 & 13 & $33.3 \pm 17.6$ & $9.9 \pm 1.6$ & 4 & 2 & 4 & 4 & 1 & 1 & 1 & 2 \\
Control & 22 & 9 & 13 & $35.7 \pm 13.9$ & $9.5 \pm 2.0$ & 6 & 3 & 5 & 3 & 2 & 1 & 1 & 1 \\
\hline
\end{tabular}

Table II. Clinical effects of the two groups.

\begin{tabular}{lccccccc}
\hline \multirow{2}{*}{ Group } & Case & Treatment times & Superior (case) & Good (case) & Moderate (case) & Poor (case) & Effective rate (\%) \\
\cline { 4 - 8 } & & & & & \multicolumn{2}{c}{ Clinical curative effect evaluation } \\
Treatment & 19 & $1.84 \pm 0.83^{\mathrm{a}}$ & 13 & 5 & 1 & 0 & $94.7^{\mathrm{b}}$ \\
Control & 22 & $2.55 \pm 0.96$ & 8 & 10 & 4 & 0 & 81.8 \\
\hline
\end{tabular}

${ }^{\mathrm{a}} \mathrm{P}<0.05$, compared with the control group on treatment times; ${ }^{\mathrm{b}} \mathrm{P}<0.05$, compared with the control group on effective rate.

During the operation, pulmonary arterial pressure was examined. With use of no. 7 butterfly needle a puncture was made into the deep part of the tumor cavity through the skin, then contrast agent was injected (30\% meglumine diatrizoate) after blood reflux and reflux venous development was observed. DSA was performed according to the recorded dose of contrast agent and then rapidly absolute ethyl alcohol was injected several times at rate of $0.5 \mathrm{ml} / \mathrm{sec}$. After $15 \mathrm{~min}$, another DSA was performed, with injection pressure at 13.3-26.7 $\mathrm{kPa}$, each injection for 0.5-1.5 ml. This was repeated once again 2-5 min later. The injection amount of absolute ethyl alcohol was about $1 / 2-1 / 3$ of the contrast agent. The maximum dose for one injection was not over $1 \mathrm{ml} / \mathrm{kg}$. After injection, the patients' blood pressure and heart rate were observed. The patients with $0.5 \mathrm{ml} / \mathrm{kg}$ injection, were monitored for their blood pressure and the urine volume. Equilibrium liquid, dexamethasone, sodium bicarbonate and proper amount of antibiotics were injected i.v. to prevent the occurrence of edema, renal function damage, infection and other adverse reactions.

Patients in the treatment group were treated by RF before anhydrous ethanol injection sclerotherapy. RF was performed under local anesthesia. R-2000BD1 bipolar radiofrequency thermal coagulation was applied with the therapy apparatus (Beijing BNS Medical Co., Ltd., Beijing, China). The electrode needle was punctured into the tumor through the head and neck skin or oral mucosa. The area of abundant blood supply was identified according to the impedance value of the puncture region detected by the computer. After the needle core was removed, blood return could be seen. Then RF was performed in this region. The RF output power was 30-40 W, and the total heating coagulation of a single lesion lasted for 10-15 min. Multiple heat coagulation could be carried out and each heat coagulation area had some overlap. The normal tissues $(0.5-1.0 \mathrm{~mm})$ were taken in the tumor within the treatment range, as the standard. After treatment, the RF ablation therapeutic instrument was adjusted into needle tract mode, pulled out the electrode needle, pressed to stop bleeding, and observed the changes of vital signs in the operation. Deep VMs can be treated by puncture under the guidance of color Doppler ultrasound. One month later, proper amount of anhydrous ethanol hardener was injected according to the change of tumor size. Methods referred to the control group.

Evaluation on clinical curative effects. Evaluation was made according to the grading standard of Achauer et al (6): level I (poor), tumor shrank by $0-25 \%$; level II (moderate), tumor shrank by $26-50 \%$; level III (good), tumor shrank by $51-75 \%$; level IV (superior), tumor shrank by 76-100\%. Level III and IV were considered to be effective; level I and II were considered to be non-effective. The treatment was continued with the aforesaid method if it was effective. The general and side adverse reactions of the patients were closely monitored during treatment. All patients had local swelling and pain after operation, which was relieved within 3-5 days. It was considered to be a reaction of hardening agent or physical treatment mechanism. Thus, it was not included as an adverse reaction.

Statistical processing. SPSS 17.0 (Chicago, IL, USA) was used for statistical analysis. Measurement data were presented as mean \pm standard deviation and the t-test was used for comparisons. The $\chi^{2}$ test was used for comparison of countable data. $\mathrm{P}<0.05$ was considered to be statistically significant.

\section{Results}

Curative effects. The curative effects of the two groups are shown in Table II. In the treatment group the treatment times were significantly less than those of the control group $(\mathrm{P}<0.05)$. The results of MRI examinations were compared before treatment and 8 weeks after the last treatment to obtain the final efficacy. It was found that the effective rate of the treatment group was significantly better than the control group $(\mathrm{P}<0.05)$. Figs. 1-3 show typical cases. 

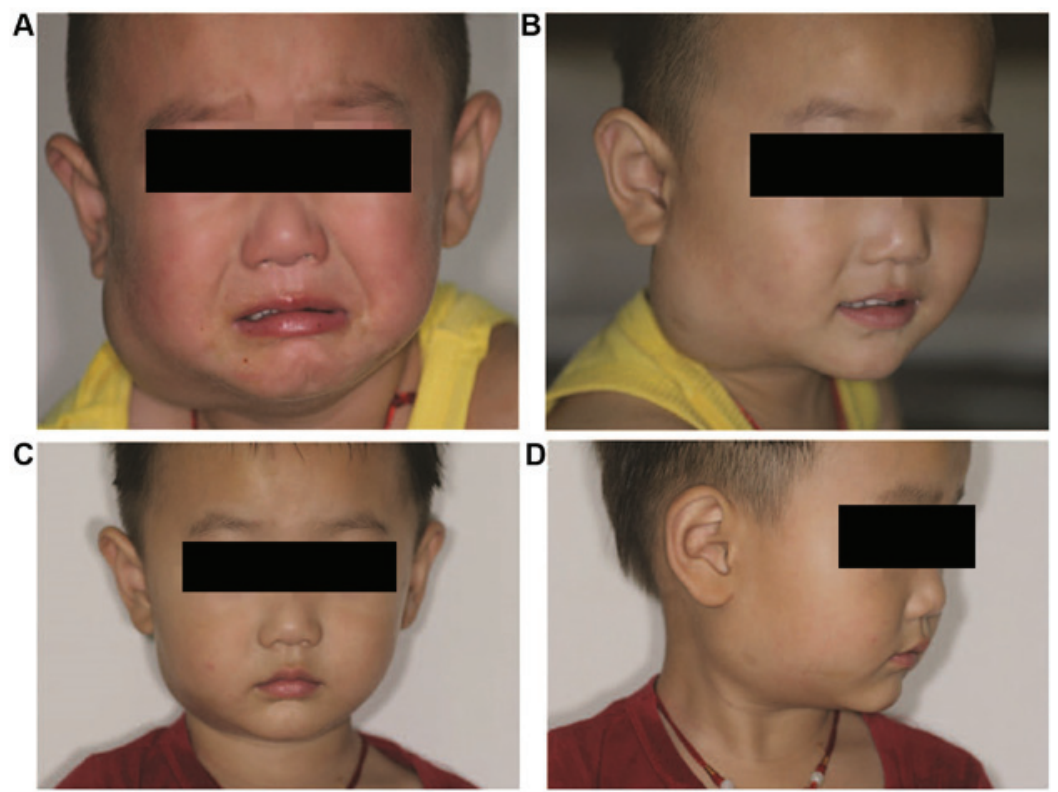

Figure 1. Venous malformation located in the right parotid gland region; (A) frontal view pre-treatment. (B) Right lateral view pre-treatment. (C) Frontal view after radiofrequency ablation combined with two times DSA-guided anhydrous ethanol injection sclerotherapy. (D) Right lateral view after treatment. DSA, digital subtraction angiography.
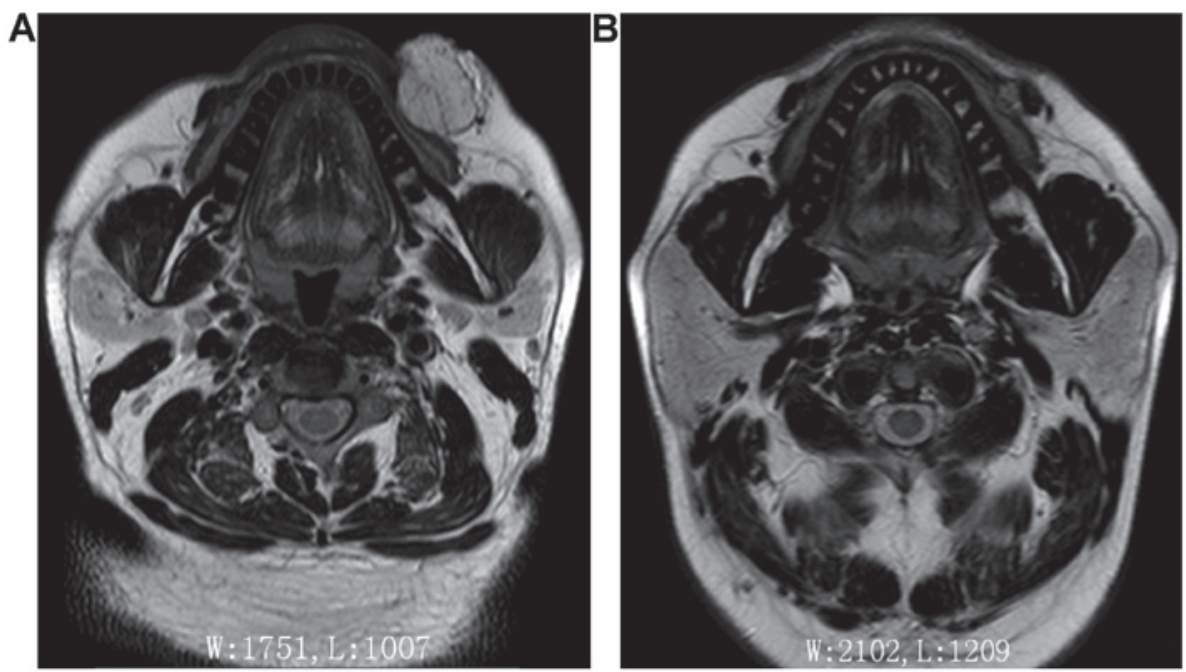

Figure 2. Comparison between pre-treatment (A) and post-treatment (B) MRI scan in patient with venous malformation of the left facial region.
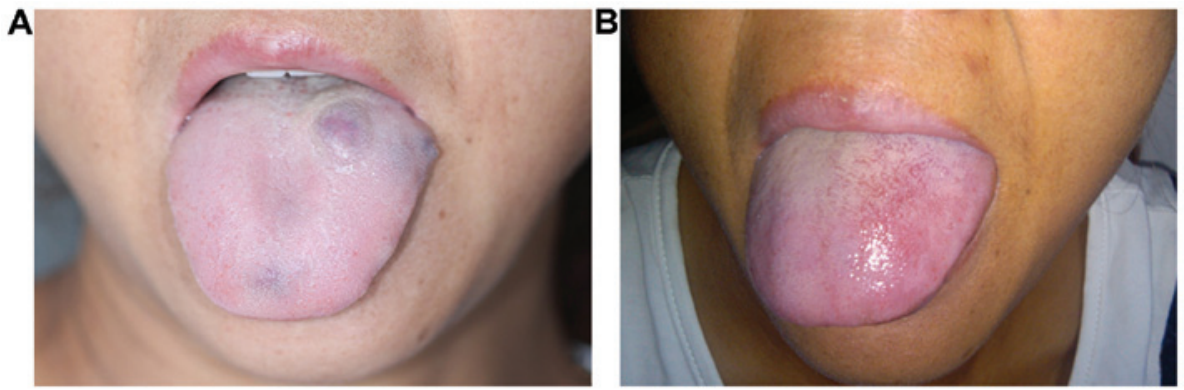

Figure 3. Venous malformation located in dorsum; (A) pre-treatment. (B) after radiofrequency ablation combined with 1 times DSA-guided anhydrous ethanol injection sclerotherapy, the lesion was involuted. DSA, digital subtraction angiography.

Complications. Throughout the course of treatment, a total of 8 patients had local mucosa or skin necrosis, which healed after local dressing. In the treatment group, 2 patients had bleeding in puncture point, but were well controlled after pressing; 
Table III. The post-treatment reaction and complication of the two groups.

\begin{tabular}{lll}
\hline Complication & $\begin{array}{c}\text { Treatment group } \\
(\mathrm{n}=19)(\%)\end{array}$ & $\begin{array}{c}\text { Control group } \\
(\mathrm{n}=22)(\%)\end{array}$ \\
\hline Fever & $0(0)$ & $1(4.55)$ \\
Nausea/vomiting & $0(0)$ & $1(4.55)$ \\
Skin, mucosal necrosis & $2(10.53)$ & $6(27.27)$ \\
Hemoglobinuria & $0(0)$ & $1(4.55)$ \\
Facioplegia & $1(5.26)$ & $2(9.09)$ \\
Hemorrhage & $2(10.53)$ & $0(0)$
\end{tabular}

Under $\chi^{2}$ examination, differences between the two groups on the above complications were not statistically significant $(\mathrm{P}>0.05)$.

1 patient with pharyngeal VM had fever, accompanied with nausea and vomiting, and was relieved after expectant treatment. In the control group, one patient had hemoglobinuria, which disappeared 3 days later, and no renal function damage was found during the follow-up period; 3 patients with deep $\mathrm{VM}$ in parotid region, after the second sclerotherapy, had the symptoms of disappearing forehead lines and shallowing nasolabial groove. After neurotrophic drug treatment and functional treatment, 2 patients returned to normal while the other case has failed to recover after 11-month follow-up. Table III provides details regarding complications.

\section{Discussion}

In recent years, sclerotherapy has replaced surgical treatment and has become the major treatment method for VMs (7). The most commonly used hardeners in China include pingyangmycin, anhydrous ethanol, lauromacrogol, and recently, it has been reported that bleomycin has positive effects in the treatment of VMs. For the superficial VM with limited lesion (8), intra-tumoral injection of the above hardeners could achieve very good curative effects. However, the patients whose lesions occur in the head and neck featured by complicated anatomic structure are usually dispersed high reflux VM. For this type of patients, the comprehensive sequence therapy that focuses on DSA-guided anhydrous ethanol injection sclerotherapy is usually considered to be quite effective (9-11). Anhydrous ethanol is a powerful hardening agent that could denature the hemoglobin by damaging the vascular endothelial cells and form permanent thrombosis and fibrosis. It has been confirmed that anhydrous ethanol has better embolization effects on the VM reflux vein and cavity than other hardening agents (12). Although its curative effects are significant, its damage on the surrounding tissues cannot be ignored. It is always accompanied with serious complications, including pulmonary embolism, hemoglobinuria, renal failure, upper respiratory tract obstruction and permanent facial paralysis $(13,14)$. A basic study has reported that ethanol can disrupt the formation of motor root myelin sheath of the facial nerves and may have a permanent effect on the nervous system (15).

$\mathrm{RF}$ ablation is a type of physical therapy. It has been confirmed to be effective and safe in the treatment of vascular disease. Therefore, it has been considered to be quite suitable for the treatment of giant vascular lesions $(d>5 \mathrm{~cm})(8,16)$. The application of pure RF in head and neck VM has been previously reported $(17,18)$. A retrospective study found that applying RF in large scale VM could significantly reduce the dosage of traditional hardening agent, and also effectively reduce the bleeding during operation (17). However, to the best of our knowledge, no report on the combined use of RF and traditional treatment method is available. In the current study, we applied RF and DSA-guided anhydrous ethanol injection sclerotherapy in treatment with the high-return flow VM. According to the literature, the effective rate of pure hardener on VM was 75-95\% (19). In our study, the effective rate of the control group was $81.8 \%$ while the effective rate of the treatment group was $94.7 \%$, significantly higher than the control group. Additionally, the treatment times of the treatment group were less than those of the control group.

Selecting the appropriate dose of anhydrous ethanol according to the volume of tumor is significant for preventing side effects $(19,20)$. Excessive anhydrous ethanol injection may lead the anhydrous ethanol flow back to the supplying artery, and result in the necrosis of skin and mucous membrane. Applying RF ablation to reduce the volume of tumor, then combining with anhydrous ethanol injection may reduce the number and dosage of injection, and decrease the risks of side reactions. In the present study, the incidence of adverse reaction of the control group reached $50 \%$, significantly higher than that of the treatment group, especially in the parotid region. The bipolar radiofrequency temperature control and thermal coagulation therapy apparatus can preliminarily determine the location of puncture needle by measuring the impedance of the needle, thereby avoiding damage to the nerve tissue (impedance, 400-550) (21). Thus, the particular performance of RF may avoid damaging the facial nerve and reducing the occurrence of facial paralysis. However, these findings remain to be corroborated in larger cohorts.

The results of the present study have shown that RF combined anhydrous ethanol injection sclerotherapy cannot only improve the effect of local treatment, but also reduce the incidence of adverse reactions. However, prior to treatment, attention should be paid to its indications and contraindications: i) for the VM in parotid region involving facial nerve, attention must be paid to the depth and direction of RF puncture needle insertion. Cautious control of the temperature to prevent the occurrence of facial paralysis. While injecting anhydrous ethanol into this region, we shall embolize the reflux vein as far as possible and eliminate the tumor cavity by heat coagulation; ii) for the VM in pharynx oralis, attention should be paid to the prevention of the upper airway obstruction resulting from tissue swelling and necrosis; and iii) for the VM in eyelid, attention should be paid to the temperature of heat coagulation. The temperature must not be extremely high, or it may injury the eyeball. In conclusion, RF combined DSA-guided anhydrous ethanol injection sclerotherapy provides a new way for treatment of high-return flow VM.

\section{Acknowledgements}

The present study was supported by the Jiangsu Province Special Program of Medical Science (BL2013008). 


\section{References}

1. Dubois $\mathbf{J}$ and Garel L: Imaging and therapeutic approach of hemangiomas and vascular malformations in the pediatric age group. Pediatr Radiol 29: 879-893, 1999.

2. Hou R, Guo J, Hu K, Yang Y, Wang L, Kong L, Liu G and Lei D: A clinical study of ultrasound-guided intralesional injection of bleomycin A5 on venous malformation in cervical-facial region in China. J Vasc Surg 51: 940-945, 2010.

3. Dompmartin A, Vikkula M and Boon LM: Venous malformation: Update on aetiopathogenesis, diagnosis and management. Phlebology 25: 224-235, 2010.

4. Yakes WF, Gibson MD and Parker SH: Alcohol embolotherapy of vascular malformations. Semin Interv Cardiol 6: 146-161, 1989.

5. Puig S, Casati B, Staudenherz A and Paya K: Vascular low-flow malformations in children: current concepts for classification, diagnosis and therapy. Eur J Radiol 53: 35-45, 2005.

6. Achauer BM, Chang CJ and Vander Kam VM: Management of hemangioma of infancy: review of 245 patients. Plast Reconstr Surg 99: 1301-1308, 1997.

7. Greene AK and Alomari AI: Management of venous malformations. Clin Plast Surg 38: 83-93, 2011.

8. Garg S, Kumar S and Singh YB: Intralesional radiofrequency in venous malformations. Br J Oral Maxillofac Surg 53: 213-216, 2015.

9. Eivazi B and Werner JA: Venous and arteriovenous malformations in the head and neck region. Therapeutic options and challenges. HNO 62: 19-24, 2014 (In German).

10. Judith N, Ulrike E, Siegmar R, Matthias N and Jürgen H: Current concepts in diagnosis and treatment of venous malformations. J Craniomaxillofac Surg 42: 1300-1304, 2014.

11. Meng J, Zhuang QW, Gu QP, Zhang J, Li ZP and Si YM: Digital subtraction angiography (DSA) guided sequential sclerotherapy for maxillofacial vein malformation. Eur Rev Med Pharmacol Sci 18: 1709-1712, 2014.

12. Goyal M, Causer PA and Armstrong D: Venous vascular malformations in pediatric patients: comparison of results of alcohol sclerotherapy with proposed MR imaging classification. Radiology 223: 639-644, 2002.
13. Lee KB, Kim DI, Oh SK, Do YS, Kim KH and Kim YW: Incidence of soft tissue injury and neuropathy after embolo/sclerotherapy for congenital vascular malformation. J Vasc Surg 48: 1286-1291, 2008.

14. Odeyinde SO, Kangesu L and Badran M: Sclerotherapy for vascular malformations: Complications and a review of techniques to avoid them. J Plast Reconstr Aesthet Surg 66: 215-223, 2013.

15. Komatsu S, Sasaki Y and Shiota K: A quantitative study of the facial nerve in mice prenatally exposed to ethanol. Congenit Anom (Kyoto) 43: 41-45, 2003

16. Zou H, Yan J, Wu YX, Ou X, Li XW, Xia F, Ma KS and Bie P: The new technology of enhanced radiofrequency ablation is safe and effective for treating giant hepatic hemangioma. Zhonghua Gan Zang Bing Za Zhi 20: 261-265, 2012 (In Chinese).

17. Kim AH, Ko HK, Won JY and Lee DY: Percutaneous radiofrequency ablation: A novel treatment of facial venous malformation. J Vasc Surg 50: 424-427, 2009.

18. Civelek S, Sayin I, Ercan I, Cakir BO and Turgut S: Bipolar radiofrequency-induced interstitial thermoablation for oral cavity vascular malformations: Preliminary results in a series of 5 children. Ear Nose Throat J 91: 488-492, 2012.

19. Lee CH and Chen SG: Direct percutaneous ethanol instillation for treatment of venous malformation in the face and neck. Br J Plast Surg 58: 1073-1078, 2005.

20. Liu Y, Liu D, Wang Y, Zhang W and Zhao F: Clinical study of sclerotherapy of maxillofacial venous malformation using absolute ethanol and pingyangmycin. J Oral Maxillofac Surg 67: 98-104, 2009.

21. Oturai AB, Jensen K, Eriksen J and Madsen F: Neurosurgery for trigeminal neuralgia: comparison of alcohol block, neurectomy, and radiofrequency coagulation. Clin J Pain 12: 311-315, 1996 